\title{
Que disent les médecins généralistes de leurs besoins de formation en gynécologie?
}

\author{
Gwénola LEVASSEUR*, Catherine BAGOT*, Charles HONNORAT*
}

\begin{abstract}
Résumécontexte: En F rance, la formation des médecins généralistes en gynécologie est un nouvel enjeu pour le système de santé. Elle doit permettre de compenser la pénurie annoncée des gynécologues, d'assurer un accès équitable au dépistage et aux soins. Objectifs : - Analyser ce que disent les médecins généralistes de leurs besoins de formation. - En décrire les déterminants. M éthodes :- Enquête qualitative par entretiens auprès de 21 médecins géné ralistes. - Analyse du contenu de ces entretiens. Résultats : U ne typologie peut être proposée. 1. un premier groupe de médecinsn'exprime pas de besoins de formation : par manque detemps, d'intérêt personnel, de demandes ou parce qu'ils estiment avoir les compétences suffisantes 2 . un deuxième groupe exprime très vivement des besoins de formation : il sagit de médecins exerçant en milieu rural. 3. un troi sième groupe se dédare très sensi ble à l'évolution de la demande des femmes et l'expression de leurs besoins est directement liée à l'évolution de leur activité de gyné cologie. 4. le dernier groupe est constitué de médecins qui n'expriment pas de besoins et qui se déclarent essentielle ment sensibles à l'aspect convivial de la FM C. Conclusion, perspectives Les besoins de formation des généralistes en gynécologie s'inscrivent dans un contexte très dépendant de la demande des femmes et des contraintes d'exercice. Le principal déterminant de l'expression de besoins de formation est l'évolution de la demande.
\end{abstract}

\section{Mots CléSFormation continue ; besoins de formation ; médecine générale ; gynécologie.}

Summary context:In France, the training of GPs in gynaecology is a new challenge for the health system. The main concern is to compensate for the announced shortage of gynaecologists and to ensure equity in scree ning and care. Objectives: - to analyse what G Ps say about their educational needs. - to describe their determinants. M ethods:- Q ualitative survey (21 interviews with GPs) - Content analysis Results: A typology in four groups is proposed: - The first is composed of G Ps who express no training needs : because of lack of time, personal interest or adequate skills. - The second express high needs for continuous training. These are G Ps working in rural areas. - The third is aware of the evolution of women's request. The educational needs expressed are clearly linked with the level of their activity in this field. - The fourth is composed of GPs who find the conge nial aspects of the training appealing. Conclusion:G PS training needs in gynaecology depend mainly on women's demands and on the conditions of practice. The main determinant of the expression of their educational needsis the evolution of the demand.

Keywordsc ontinuing medical education; educational needs; general practice; gynaecology.

Pédagogie M édicale $2002 ; 3: 19-24$

* Département de Médecine Générale - URR de Médecine - Rennes

Correspondance : Gwénola Levasseur - 2 Avenue du Pr Léon Bernard - CS 34317, 35043 Rennes Cedex - Tel : $33+223$ 234575 - Fax : 33 + 299541396 - mailto:gwenolalevasseur@wanadoo.fr 


\section{Recherche et Perspectives}

\section{Contexte}

Le système de soins français se distingue des autres systèmes européens par les principes d'un paiement à l'acte et d'un libre accès des assurés aux praticiens généralistes ou spécialistes. Chacun peut consulter qui il veut, quand il le désire Ce qui était l'utopie des débuts de la Sécurité Sociale est devenu possible grâce au développement de l'État providence, au progrès médical et à sa médiatisation qui ont modelé le rapport de la population à la santé. La santé est devenue une valeur, une norme, un droit à être non seulement soigné mais guéri, un droit à la sécurité. $A$ quoi s'ajoute la notion de liberté (pour le patient et pour le médecin) jugée fondamentale1.

$D$ ans ce contexte, une pénurie de gynécologues est annoncée. Trois phénomènes y concourent : l'arrêt de la formation à la gynécologie médicale par voie de CES (certificat d'études spéciales) en 1984, la féminisation et le vieil lissement de cette catégorie de spécialistes dont les effectifs stagnent (cessations d'activité très partiellement compensées par les nouveaux venus à la spécialité). Aujourd'hui, on dénombre en France un gynécologue pour 3700 femmes. D es habitudes ont été prises : selon une enquête de la Sofres (Société française d'études et de sondages) menée en janvier 1998 pour la Fédération nationale des collèges de gynécologie médicale (échantillon de 1000 personnes de plus de 18 ans), $60 \%$ femmes interrogées consultent leur gynécologue au moinsune foispar an, pour $93 \%$ d'entreelles le dépistage et la prévention des cancers sont les premiers motifs de consultation. Cette situation est très spécifique à la France: dans de nombreux pays européens, la gynécologie « de base » est assurée par le médecin généraliste. «II n'y aura plus de gynécologues médicaux dans vingtcinq ans: (...) le suivi gynécologique des femmes reviendra alors aux médecins généralistes » 3 .

La formation des médecins généralistes en gynécologie apparaît donc comme un double enjeu à la fois pour le système de santé français et pour le segment professionnel*; elle doit permettre de compenser la pénurieannoncée des gynécol ogues médicaux et assurer un accès équitable au dépistage et aux soins dans un contexte de réduction des dépenses de santé.

Franck Bidault ${ }^{4}$ a réalisé un travail sur les besoins de formation des résidents de médecine générale dans un service universitaire de gynécologie. II s'agissait d'une approche très normative 5 à partir des données de la litté rature validées par les universitaires. N ous nous sommes quant à nous, intéressés aux besoins de formations des médecins généralistes tels qu'ils les ressentent quelques années après leur installation.

\section{Objectifs}

L'étude de nature exploratoire se fixait deux objectifs:

- analyser ce que disent les médecins général istes de leurs besoins ressentis de formation ;

- décrire les déterminants de l'expression de leurs besoins.

\section{Méthodes}

\section{Enquête qualitative par entretiens à usage exploratoire ${ }^{6}$ auprès de 21 médecins généra- listes d'lle et Vilaine.}

Ce type d'enquête est une démarche destinée à explorer comment une question se pose dans une population donnéé. La question de la représentativité statistique ne se pose pas : il sagit de constituer un échantillon diversifiéà partir de variables supposées a priori jouer un rôleimportant dans la structuration des discours. Les critères retenus ont éé la répartition géographique dans tout le départe ment d'I lle et Vilaine, autant d'hommes que de femmes (11 et 10), de type d'exercice rural, semi-rural ou urbain, et une ancienneté d'installation d'au moins quatre ans. L'échantillon nécessaireà la réalisation d'une telle enquête

\section{Guide d'entretien}

\section{Consigne d'ouverture}

«Pouvez-vous me parler des besoins de formation que vous ressentez dans votre pratique de la gynécologie?»

\section{Thèmes}

- L'activité de gynécologie (population de femmes suivies, leurs demandes).

- Les questions en gynécologie (connaissances, gestes techniques, situations difficiles à gérer). - La formation initiale et continue (description, besoins, formation souhaitée).

\footnotetext{
* Le concept de segment professionnel a été défini par Anselm Strauss.
} 
est plus réduit que celui d'une enquête par questionnaire dans la mesure où les informations issues des entretiens sont validées par le contexte et non par leur probabilité d'occurrence. La réduction relative de l'échantillon tient donc au statut de l'information retenue.

Le nombre d'entretiens à réal iser dépend du thème de l'enquête, de la diversité supposée des attitudes par rapport au thème, du type d'enquête (exploratoire, principale ou complémentaire), du type d'analyse projeté et des moyens dont nous disposons. Par ailleurs, à partir d'un certain nombre d'entretiens les informations recueillies sont redondantes : il y a «saturation » de l'information. Leguide d'entretien a été élaboréà partir de la description des objectifs éducatifs normatifs décrits par F. Bidault dans sa thèse'.

D ans la préparation d'une enquête quantitative ce type d'entretien est un outil privilégié puisqu'il informe sur le vocabulaire et les opinions. L'analyse du contenu des entretiens permet d'élaborer un questionnaire destinéà la réalisation d'une étude quantitative.

\section{Analysedes données*}

D e nombreuses méthodes permettent l'analyse des données recueillies par les entretien $s^{6}$, c'est-à-dire de leur contenu. C ertaines sont accessibles sans l'outil informatique comme, par exemple, l'anal yse thématique ${ }^{8}$. $D$ 'autres ont recours à des procédés statistiques plus ou moins complexes. Celle qui est utilisée dans ce travail est la plus usitée : I'analyse factorielle des correspondances'. Les sociologues I'ont empruntéaux psychologuesqui euxmêmel'ont prise aux linguistes. Cette analyse a pour but, à partir de l'idée de corrélation, de découvrir des relations d'interdépendance entre différents facteurs. Elle permet de traiter de très grands tableaux de contingence et de les représenter visuellement sous une forme géométrique (graphe). Les résultats sont donnés par la lecture des regroupements de variables sur le graphe. Ces regroupements correspondent aux associations préférentielles et quantitativement mesurables entre les variables. L'extraction des résumés des textes sous-jacents se fait de façon automatisée.

L'intérêt de cette méthode est de prendre en compte l'exhaustivité des données (caractéristiques des médecins, entretiens et l'ensemble des thèmes abordés).

\section{Résultats}

Les médecins interviewés se répartissent en quatre groupes.

\section{Le premier groupe nexprime pas de besoins de formation}

Ces médecins n'ont pas de caractéristiques particulières par rapport aux critères de sélection retenus. Dans ce groupe la situation est contrastée : on trouve à la fois des médecins qui ne font pas ou peu de gynécologie et des médecins qui s'estiment suffisamment formés à cette activité.

Chez les premiers il y a des généralistes que les femmes consultent peu pour leur suivi «La gynécologie, ça n'est pas la majeure partie de mon activité »; " je ne suis que les quelques femmes qui me demandent de le faire » et des généralistes que cette activité n'intéresse pas « par choix personnel, parce que ça ne me passionne pas », « la gynécologie c'et pas vraiment mon truc ». II s'ensuit une absence de besoins de formation.

II peut aussi s'agir de médecins qui s'estiment suffisamment formés à cette activité et ne ressentent pas de besoins « Je n'ai pas de problèmes, je n'ai pas de questions (...), non vraiment je ne vois pas »; « ce dont j'ai besoin je sais le faire : je ne vois pas quelle formation pourrait me servir » ou de médecins qui manquent de temps, soit du temps (réel ou imaginé) nécessaire à la réalisation d'un acte de gynécologie « les consultations de gynécologie prennent beaucoup de temps et ça ne mintéresse pas » soit du temps nécessaire pour suivre une formation « jefais beaucoup de gynécologie, mais je n'ai pas besoin de formation, d'ailleurs jen'en aurais pas le temps... ».

\section{Le deuxième groupe exprime très vivement des besoins de fomation}

Ces médecins exercent en milieu rural, à distance des spé cialistes, et sont donc très sollicités pour le suivi gynécologique de leurs patientes. " 0 n voit presque toutes les femmes, parcequ'on es à la campagne» ; "J'ai des patientes qui vont voir leur gynécologue, j'ai aussi des patientes que je ne vois quasiment que pour la gynécologie».

Les besoins qu'ils expriment sont d'abord liés à des difficultés ressenties. Difficultés de communication ou relationnelles «Je souhaiterais surtout une formation psycholo-

\footnotetext{
* Traitement et analyse effectués par Michel Kerbaol et Jean-Yves Bansard, statisticiens INSERM (Institut national de la santé et des sciences médicales).
} 


\section{Recherche et Perspectives}

gique, quand on a un problème au niveau médical, on passe la main, la difficulté, c'est plutôt au niveau psychologique ». Les difficultés de la gestion des motifs multiples lors des consultations est soulignée: "L Le seul problème à gérer en médecine générale, c'est lors d'une seule consultation la multiplication des problèmes.. j'ai quasiment à chaque consultation gynécologiqueun autre problème qui segreffe dessus». $M$ ais aussi les exigences des patientes: " 30 à $50 \%$ des renouvellements de pilule $₫$ font dans le couloir (...) la demande se fait en général quand on a fini d'examiner es gamins et qu'on sapprête à partir : « je voudrais ma pilule.. »; « elles ont leur gynéco, leur pédiatre... et le médecin généraliste après $19 \mathrm{~h}$ le soir ! 》.

Les médecins de ce groupe souhaitent une formation très adaptée à leur pratique, qu'il sagisse de gestes techniques ou de thérapeutiques. Les besoins concernent très majoritairement les traitements hormonaux (contraception orale, mise en route d'un traitement hormonal substitutif et son suivi). "J'aimerais une information daire sur la contraception estroprogestative.. j'aimerais saisir les nuances qu'il y a, les différents types de progestatifs et les différents dosages, mieux cibler les choses. C'et toujours ce qui me manque, l'information objective... ».

\section{Le troisième groupe se déclare très sensible à l'évolution de la demande des femmes}

Ces médecins exercent tous en milieu urbain ou semiurbain, donc à proximité des spécialistes. L'évolution de leur activité de gynécologie est très contrastée, l'expression de leurs besoins est directement liée à l'évolution de leur activité

Certains d'entre eux regrettent que la demande diminue. La plupart de leurs patientes sont maintenant suivies par un (ou plus souvent une) gynécologue médical et ils estiment en conséquence qu'ils ont désormais des besoins de formation limités. "Les besoins ont beaucoup évolué au cours des années. Je faisais beau coup de gynéco-obstétrique (...). J'ai aujourd'hui une pratique très ponctuelle, très modé rée et donc des besoins très restreints, avec la demande qui diminue, les questions sont moins fréquentes ; ; "Je ne vois pas bien l'intérêt d'apprendre des gestes techniques qui ne me servi ront pas dans ma pratique ", D'autres au contraire, sont de plus en plus sollicités dans le suivi gynécologique de leurs patientes et lient cette augmentation d'activité au manque de disponibilité des gynécologues.

«Presque toutes les patientes sont mai ntenant suivies par un gynéco, le problème c'est leur manque de disponibilité » ;
« pour adapter le trai tement, c'est vers nous qu'elles se tournent.(...) d'autant plus que les gynécologues ne sont pas souvent di sponibles, on se débrouille ». La compétence du généraliste dans ce domaine crée aussi la demande «J efais beau coup de gynécol ogie (...) je suis la seule au cabinet à poser les stérilets. II arrive très souvent que mes collègues m'adressent une deleurs patientes pour la posed'un stérilet. Je fais beaucoup de prescriptions de pilule, de suivis de grossesse et de plus en plus car les gynécol ogues manquent de temps ». Q ue leur activité augmente ou diminue, les modalités mêmes de l'exercice évoluent ; de plus en plus facilement, les médecinsn'hésitent pasà demander des examens complémentaires (proximité des laboratoires, des radiologues) pour «se rassurer et rassurer leurs patientes ». D ès que les problèmes dépassent le champ de leurs connaissances, ils savent rapidement passer la main au spécialiste « D ans toutes les situations plus compliquées, j'ai recours aux spécialistes». Beaucoup dénoncent «lemalaise qui existe actuellement entre les général istes et les gynécol ogues » tout en revendiquant une place pour le médecin généraliste dans le suivi des femmes «nous ne devrions pas être en concurrencemais complémentai res» et en soulignant les habitudes prises en ce domaine " Lesfemmes vont de plus en plusvoir directement le gynécologue, parce qu'on leur a donnél'habitude d'être suivie par un spécialiste; c'et de là que vient le malaisequi exi ste actuellement entreles médecins généralistes et lesgynécologues.. Le suivi gynécol ogique relèvetout à fait de la médecine générale; on a l'impression d'une chasse gardée, c'est vraiment dommage! »

Lorsque des besoins de formation sont exprimés, la demande est avant tout celle d'une formation adaptée à leur pratique de la gynécologie : savoir répondre aux questions de leurs patientes (antécédents, facteurs de risque, etc.), pouvoir soulager les petits maux du quotidien (allaitements difficiles, bouffées de chaleur, etc.) "J'ai surtout besoin de choses pratiques Je n'attends pas de recette mirade, mais des peits trucs pour améliorer la prise en charge de mes patientes». " ce ne sont pastoujours de gros problèmes, des choses qui rendent service, pour régler des petits problèmes, le changement de pilule s la première n'est pas bien supportée».

Comme dans le groupe précédent le besoin d'une formation psychologique, d'une formation à l'écoute et à la communication est exprimé. "J'ai des difficultés face à la demande d'avortement, c'est une stuation relationnelle difficile»; " J e souhaiterais une formation à l'écoute en gynécologie... U neformation complémentai re serait souhaitable en communication, écoute»; " II faudrait une formation à la 
relation, surtout pour les méded ns hommes qui suivent des femmes sur le plan gynécologique».

\section{Le dernier groupe est constitué de médecins qui rexpriment pas de besoins et qui se déclarent essentiellement sensibles à l'aspect convivial de la FMC.}

Une FM C est l'occasion pour eux de rencontrer leurscorrespondants; ils sont sensibles à l'aspect convivial de la soirée... «C'est l'occasion deretrouver les confrères, d'échanger nos points de vue; ça permet demieux connaître nos correspondants (...) quelque chose de convi vial (...), on ne cherche pas à avoir des connaissances encydopédiques, mais quelquechose d'adapté, de moderne»,

\section{Discussion}

Une telle enquête n'a nullement la prétention de définir les besoins de formation des général istes, mais elle permet d'en approcher la dimension personnelle, les représentations que se font les médecins de leurs besoins de formation en gynécologie et les motivations qui les sous-tendent. Elle permet également de cerner les questions problématiques et de proposer quelques hypothèses. Les commentaires suivants veulent stimuler la réflexion. II convient de rappeler que les résultats restent fragiles parce que fondés sur un nombre limité d'entretiens, mais qu'ils n'en apportent pas moins une information d'un grand intérêt. II conviendra de valider ces résultats par une enquête plus large.

Le premier constat est que si les généralistes se déclarent prêts à assumer de façon occasionnelleun acte de gynécologie, tous ne souhaitent pas forcément s'engager dans un suivi régulier de leurs patientes, certains disent même n'avoir aucun intérêt pour cetyped'activité. En théorie, le fait d'avoir des actes de gynécologie à effectuer devrait dépendre de facteurs objectifs qui leur sont extérieurs : nombre de femmes dans la zone géographique d'exercice, importance numérique de la clientèle et proximité des gynécologues. En réal ité, ces divers facteurs interviennent dans le cadre d'itinéraires professionnels et sans doute personnels. D enombreux auteursont décrit lefait queles médecins choisissent et construisent leur clientèle tout autant que les patients les choisissent ${ }^{10}$. La sélection de la clientèle est en fait une relation symétrique et en la matière, «la clientèle fait le médecin et le médecin fait la clientèle ${ }^{11}$.

Comme l'avait démontré Freidson, la pratique du méde cin est avant tout une pratique d'application qui «se concentre sur des problèmes concrets et leur solution pratique » ${ }^{12}$. Et c'est vraisemblablement dans «l'expérience » du contact aux patientes, dans la résolution pratique de leurs problèmes que se fait le passage d'un état d'incertitude ou de réticence à une conduite professionnelleadaptée.

L'expression de besoins de formation en gynécologie relève, pour certains médecins, des représentations qu'ils se font de la médecine générale. C'est ainsi qu'ils réclament une place spécifique dans le suivi des patientes que leur insertion dans le quotidien et leur connaissance des contextes de vie peut faciliter. C e discours, un peu réducteur, est sans doute celui de généralistes militants. En effet, selon cet argumentaire, il suffirait de donner aux général istes un apprentissage pratique et régulièrement actualisé pour leur donner un rôle plus actif dans le suivi gynécologique des femmes...

Enfin, pour certains l'expression de besoins de formation repose sans doute sur la recherche d'un sentiment d'appartenance à un groupe ou sur un besoin de reconnaissance.

Les généralistes évoquent aussi leurs difficultés d'exercice : difficultés liées au comportement et aux attentes des patientes. La pratique des généralistes peut être plus que tout autre segment de la profession sous la dépendance de la clientèle. Un des premiers pouvoirs du patient en effet réside dans sa capacité de choisir son médecin. Le patient crée et contrôle en partie cette relation : le satisfaire est un moyen de le fidéliser ${ }^{13}$.

Un constat peut également être fait sur la perception qu'ont les médecins de leurs relations avec les patients dans le système de santé. Le recours fréquent des femmes au gynécologue illustre le prestige de la position de spécialiste auprès des profanes. Les gynécologues sont décrits par 88 \% des femmes interrogées dans l'enquête Sofres* comme plus compétents, comme ayant plus de connaissances et de pratique qu'un médecin généraliste dans le domaine du dépistage et de la prévention. Le problème clé pour les généralistes est donc celui d'un équilibre précaire entre les demandes des femmes et les offres concurrentielles des spécialistes. II s'agit là d'un problème structurel du système de santé français.

${ }^{\star}$ Op cit. 


\section{Recherche et Perspectives}

\section{Conclusion, perspectires}

Cette analyse permet de préciser les déterminants des besoins de formation actuellement ressentis par les médecins général istes français dans le domaine de la gynécologie. Ces besoins sont divers et de nature variable. IIs sont influencés par la demande des femmes et par les contraintes des conditions d'exercice. Le principal déterminant de l'expression de besoins de formation semble bien être l'engagement personnel qu'ils manifestent dans le suivi gynécologique des femmes. Puisque la clientèle fait le médecin, la demande des femmes et les modifications prévisibles de leurs habitudes pèseront vraisemblablement dans le futur d'un poids non négligeable.

L'identification des besoins de formation des médecins généralistes en gynécologie et en obstétrique passe vraisemblablement plus par l'analyse du contexte professionnel du demandeur que par la déclinaison de besoins normatifs i ssus de l'analyse de compétences « idéales ».

II ne faudrait pas non plus méconnaître le rôle des institutions. Bon nombre des besoins de formation des généralistes sont dictés par les évolutions de l'organisation du système de santé. Cependant, les modifications de leurs pratiques professionnelles peuvent dépendre de déterminantsautres quelaformation : «Q uand lebesoin exprimé justifie un changement de comportement, ce n'est pas toujours la formation du praticien qui permettra lemieux de l'obtenir. C ertaines évolutions relèvent plus certainement de la modification réglementaire ${ }^{14}$.

L'analyse du contenu des discours a permis de proposer un questionnaire qui, testé sur un groupe de 70 personnes, a objectivé sa pertinence. II fait actuellement l'objet d'une diffusion régionale. Les données recueillies permettront une approche quantitative et thématique des besoins deformation exprimés par les généralistes dans le domaine de la gynécologie.

\section{Remerciements}

A M ichel Kerbaol et Jean-Yves Bansard (Statisticiens de I'IN SERM , D épartement de Santé Publique, Université de Rennes 1) qui ont traitéles données.

A I'U nion Régionale des M édecins Libéraux de Bretagne (U RM LB) pour son soutien financier.

7. Grawitz M. M éthodes des sciences sociales. Paris : Dalloz, 1996.

8. Ghiglione R, BeauvoisJ-L, Chabrol C, Trognon A. $M$ anuel d'analyse de contenu. Paris : Armand Collin, 1985.

9. Benzécri J-P. H istoire et préhistoire de l'analyse des données. Paris: Dunod, 1982.

10. Parsons T. The sick role and the role of physician reconsidered. M ilbank M em Fund Q 1975 (summer) : 257-278.

11. Brams L, Radenac H, Carcenac M, N akache P. $M$ ilieux urbains en mutation, des problèmes de santé publique. C. Sociol. D émogr. M édic. 1972 (4) ; 107-125.

12. Friedson E. La profession médicale. Paris: Payot, 1984.

13. Friedson E. Client control and medical practice. Am J Sociology 1960 (65) : 374-382.

14. H onnorat $C$, L evasseur $G$. Q uels besoins enseigner, quel besoin d'enseigner ? Pédagogie M édicale 2001; 2(1) : 26-30. 